\title{
Output characteristics of Q-switched solid-state lasers using intracavity MEMS micromirrors
}

\author{
Ralf Bauer, Alan Paterson, Caspar Clark, Deepak Uttamchandani, and Walter Lubeigt
}

\begin{abstract}
The output behavior of a Nd:YAG solid-state laser actively Q-switched by a MEMS scanning micromirror is presented. Using a gold-coated micromirror, maximum average output powers of $50 \mathrm{~mW}$ and pulse durations as short as $120 \mathrm{~ns}$ were obtained with a dual beam output. This output pattern originates from a pulse emission when the micromirror is at an angle from the cavity axis. The temporal and spatial behavior of this laser was experimentally characterized and then modelled using a numerical simulation of the laser rate equations. Finally, prospects for power-scaling this MEMS-based Q-switch technique are demonstrated using a dielectric-coated micromirror, which led to average output powers of up to $650 \mathrm{~mW}$ and pulse energies above $40 \mu \mathrm{J}$.
\end{abstract}

Index Terms-Solid-state laser, MEMS scanning micromirror, active Q-switch, laser dynamics

\section{INTRODUCTION}

$\mathrm{I}^{\mathrm{n}}$ recent years, advances in silicon fabrication technology and in particular the development of multi-user silicon fabrication facilities have enabled the development of low-cost optical Microelectromechanical systems (MEMS) for laser applications. Most notably, scanning micromirrors have been integrated inside solid-state laser cavities as active mechanical Q-switches, enabling the generation of cavity-limited pulse durations at average powers exceeding 100mW [1]. Compared to more traditional active Q-switching techniques using acousto-optic modulators (AOM) or electro-optic modulators (EOM), this MEMS-based approach provides significant advantages in miniaturization, reduction of powerconsumption and cost. This technique particularly benefits from recent intense developments in optical MEMS generating new directions in optics [2]. Finally, the possibility of developing MEMS micromirror arrays will enable multiple, individually controlled, beam emission from the same laser gain medium impacting many applications in defense, industry and biology.

Over the last few years, several types of MEMS devices have been used for intracavity temporal laser control, such as a

Manuscript received April 1, 2014. This work was supported by the Scottish Encompass scheme.

R. Bauer, A. Paterson, D. Uttamchandani, and W. Lubeigt are with the Centre for Microsystems and Photonics, Department of Electronic and Electrical Engineering, University of Strathclyde, Glasgow, G1 1XW, UK (email: ralf.bauer@strath.ac.uk, alan.paterson.2013@uni.strath.ac.uk, d.uttamchandani@strath.ac.uk, walter.lubeigt@strath.ac.uk).

C. Clark is with Helia Photonics Ltd., Rosebank Park, Livingston, EH54 7EJ, UK (e-mail: caspar.clark@helia-photonics.com). piezoelectric polymer vibrating mirror [3], a cantilever mirror [4]-[6], a membrane mirror [7]-[9] and a scanning micromirror [1], [10], [11]. In this way, active Q-switching of single [4], [7], [10] and multiple [5], [8] fiber lasers with pulse energies in the single $\mu \mathrm{J}$ range and pulse durations as short as $20 \mathrm{~ns}$ for sub- $\mu \mathrm{J}$ pulses have been reported. Similarly single [3], [11] and multiple [1] Q-switched outputs of solid-state lasers have been demonstrated with pulse energies reaching $36 \mu \mathrm{J}$ and minimum pulse durations of $30 \mathrm{~ns}$. A combination of active and passive Q-switching of a microchip laser to achieve sub-nanosecond pulse durations has also been reported [6], as well as mode-locked pulse operation of a fiber laser using a membrane deformable mirror [9].

Theoretical models of the Q-switch laser dynamics were developed for solid-state lasers in the 1960s, e.g. by [12] or [13], with specific analytical descriptions of the temporal and spatial output of mechanical rotating mirror Q-switches shown using a rate equation approach [14] and a Fourier expansion technique of the electromagnetic field [15] shown in the 1990s. The laser rate equations based approach considers the temporal output, with a description of the intracavity optical loss increase due to the rotation of the mechanical Q-switch needed for determining the pulse timing. To our knowledge, no full temporal and spatial description of the laser dynamics using a MEMS-scale scanning mirror with bi-directional movement, membrane mirror or cantilever mirror has however been developed yet.

In this paper, a detailed analysis of the performance of a side-pumped Nd:YAG laser operating at $\lambda=1064 \mathrm{~nm}$ and incorporating a MEMS scanning micromirror as an active Qswitch is reported. This includes a description of Q-switch pulse timings and pulse durations together with the spatial laser output distribution with varying laser parameters. Additionally, the experimental results will be supported by an analytical model of the temporal output dynamics of the laser based on the rate equations.

Section II describes the MEMS scanning micromirror used as an active Q-switch element while section III presents the experimental results, showing the temporal and spatial laser output characteristics. Section IV provides an analysis of the laser behavior based on the rate equation model for determining the temporal theoretical laser behavior. Finally, section $\mathrm{V}$ details the latest power-scaling development of this technique using dielectric coatings. 


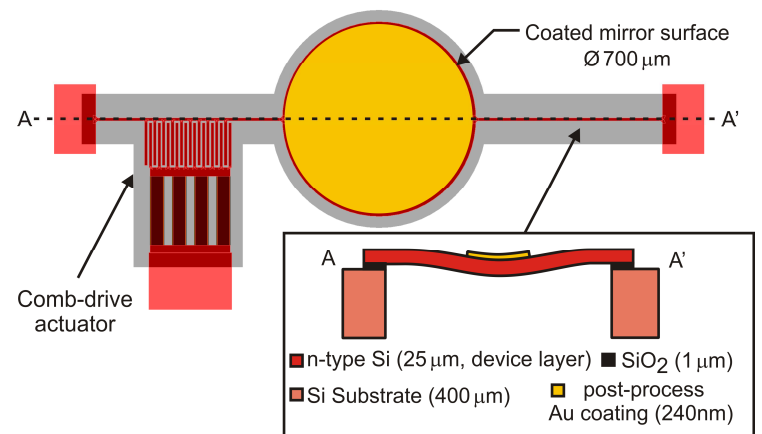

Fig. 1. Schematic layout of the MEMS scanning micromirror including the layer structure of the used SOIMUMPs fabrication process.

\section{MEMS DESIGN, FABRICATION AND CHARACTERIZATION}

\section{A. Micromirror design and fabrication}

The MEMS micromirror used in this work was fabricated using a multi-user silicon-on-insulator (SOI) commercial process [16] and has previously been described in [1]. The electrostatically-actuated scanning micromirror, made of a $25 \mu \mathrm{m}$ thick, phosphorous-doped silicon device layer and shown in Fig. 1, consisted of a circular $700 \mu \mathrm{m}$ diameter mirror suspended by two $750 \mu \mathrm{m}$ long and $12 \mu \mathrm{m}$ wide rectangular torsion springs. The length and width of the springs was chosen to induce mechanical resonance frequencies in the $\sim 10 \mathrm{kHz}$ range, while simultaneously achieving total optical scan angles (TOSA) in excess of $80^{\circ}$. The micromirror was actuated by a single-sided electrostatic comb-drive with 12 pairs of interleaved moving and fixed comb fingers. A lateral gap of $6 \mu \mathrm{m}$ was present between these $160 \mu \mathrm{m}$ long and $10 \mu \mathrm{m}$ wide fingers.

Inherent stresses in the SOI device layer, originating mainly from the inhomogeneous doping profile, led to an initial concave curvature of the mirror surface, measured using a Veeco NT1100 white light interferometer surface profiler at $0.5 \mathrm{~m}$ of radius of curvature (ROC). A scanning electron microscope image of the fabricated scanning micromirror can be seen in Fig. 2(a), including part of an array configuration which the used mirror belonged to. This array configuration and the resulting space constraints led to the use of the singlesided comb-drive. This will induce additional oscillation jitter during the mirror actuation due to the asymmetry of the structure where the actuation point of the electrostatic force is

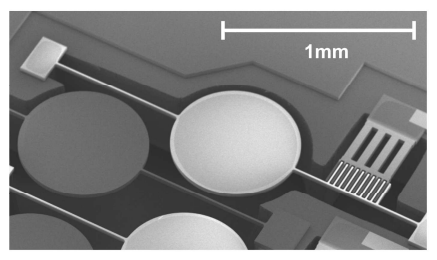

(a)

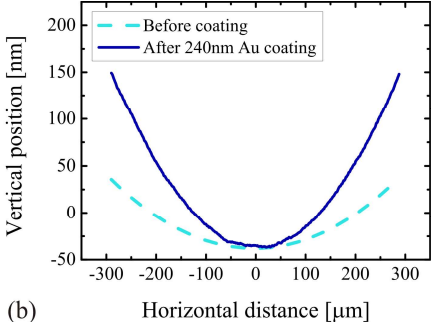

(b) off-axis. In [1], this oscillation jitter resulted in a minimal orthogonal movement during the desired resonant actuation.

As the micromirror was intended to be used inside the laser cavity, the deposition of a high reflective (HR) coating at the desired lasing wavelength was required to maintain laser conversion efficiency. Consequently, a $240 \mathrm{~nm}$ thick gold-layer was deposited on the mirror surface to ensure a reflectivity measured at $96 \%$ at $\lambda=1064 \mathrm{~nm}$ using a probe $\mathrm{Nd}: \mathrm{YVO}_{4}$ laser. Thin-film stresses originating from the difference in Coefficient of Thermal Expansion (CTE) between gold $\left(14.1 \cdot 10^{-6} \mathrm{~K}^{-1}\right)$ and silicon $\left(2.5 \cdot 10^{-6} \mathrm{~K}^{-1}\right)$ [17] led to a modification of the mirror surface curvature with a final ROC of $0.22 \mathrm{~m}$ (see Fig. 2(b)).

\section{B. Micromirror characterization}

The resonant movement shape and movement frequency were determined by the material parameters and device dimensions. A mechanical finite element method (FEM) simulation using COMSOL Multiphysics showed a resonant tilt movement, used for scanning the mirror through the laser alignment, at an eigenfrequency of $8.245 \mathrm{kHz}$ (see Fig. 3(a)), with an in-phase oscillation of the mirror and the actuating comb-drive electrodes. Further, parasitic, resonance eigenmodes of the micromirror are not excited during the tilt movement in this experiment but are located, according to the mechanical FEM simulation, at $5.88 \mathrm{kHz}$ for an in-plane bending mode, at $11.60 \mathrm{kHz}$ for an out-of-plane piston mode and at $17.26 \mathrm{kHz}$ for an in-plane rotation mode. To experimentally evaluate the resonant movement frequency and TOSA, a probe $\mathrm{HeNe}$ laser was used. The micromirror was actuated by a $200 \mathrm{~V}_{\mathrm{pp}}$ squarewave voltage signal with driving frequency values at approximately double of the mechanical resonance frequency, using a sub-harmonic driving scheme. The scanning mirror angular response to the voltage signal is shown in Fig. 3(b) for both an uncoated and coated mirror. The non-linear resonance shape observed in both cases was due to a softening behavior of the spring holding the micromirror. The uncoated mirror had a resonance peak frequency of $8.32 \mathrm{kHz}$ with a TOSA of $73^{\circ}$, with the extra mass of the coating introducing a shift of $425 \mathrm{~Hz}$ to lower resonance frequencies. The peak resonance after coating was therefore measured to be at $7.895 \mathrm{kHz}$ with a TOSA of $77^{\circ}$. The shape of the frequency response curves led
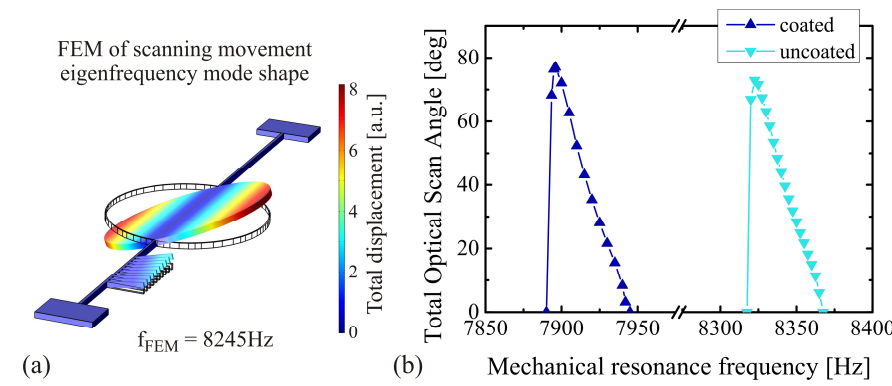

Fig. 3. (a) FEM simulation mode shape and resonance frequency for the uncoated mirror and (b) frequency response curve of the scanning movement before and after the gold coating.
Fig. 2. (a) SEM measurement of the fabricated micromirror and (b) modification of the mirror surface curvature due to the gold coating deposition. 


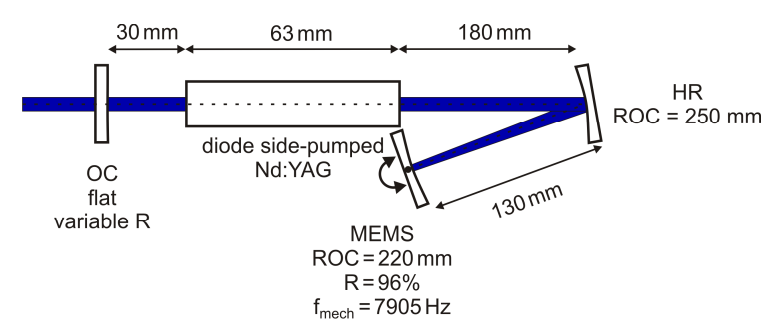

Fig. 4. Schematic of the 3-mirror laser cavity setup.

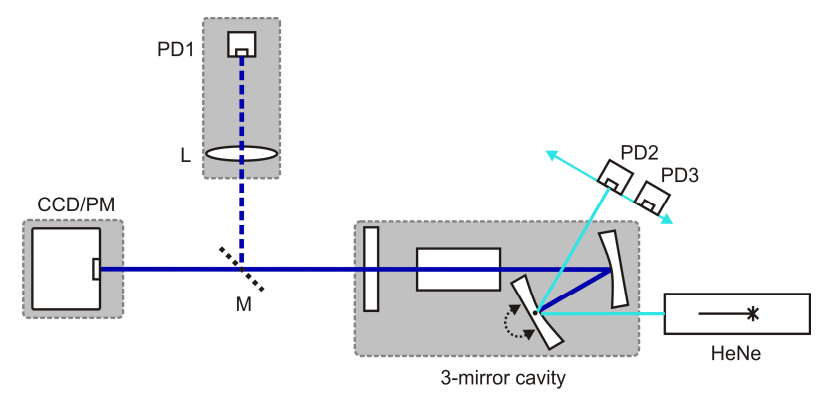

Fig. 5. Measurement schematic for temporal and spatial laser output, PD13: photodiode, L: lens, CCD: CCD camera, M: flip-able mirror, HeNe: probe laser, PM: power meter.

to a chosen mirror actuation frequency of $15.81 \mathrm{kHz}$ to avoid inducing a breakdown of the resonance movement during Qswitching, which could be caused by slight temperature imbalances and resulting frequency shifts of the resonance curve.

\section{EXPERIMENTAL LASER OUTPUT CHARACTERIZATION}

\section{A. Laser cavity and measurement setup}

To characterize the use of this micromirror as an active Qswitch device, a 3-mirror laser platform was built integrating the scanning MEMS as an end-mirror (see Fig. 4). A $63 \mathrm{~mm}$ long, diode side-pumped Nd:YAG module (Northrop Grumman RBA30-1C2) was used as a gain medium while the output coupler (OC) was placed $30 \mathrm{~mm}$ from the end-facet of the Nd:YAG crystal. A high reflective folding mirror $(\mathrm{ROC}=250 \mathrm{~mm})$ was placed $180 \mathrm{~mm}$ away from the other end facet of the gain rod, building the second cavity arm with the MEMS placed at a distance of $130 \mathrm{~mm}$. The cavity was carefully aligned to place the optical axis through the exact center of the Nd:YAG gain medium. Moreover, the MEMS micromirror was placed so that the cavity was aligned when the scanning mirror was at the midpoint position of its scanning range.

The spatial and temporal properties of the Q-switched laser output were measured using the configuration shown in Fig. 5, involving three fast $(1.5 \mathrm{GHz})$ photodiodes and a CCD camera (DataRay WinCamD-UC012-1310). Photodiode PD1 enabled the temporal measurement of the laser pulse while PD2 and PD3 provided an accurate measurement of the center point of the micromirror scanning movement and its direction. The spatial output profile of the laser output was measured at various distances from the $\mathrm{OC}$ using the $\mathrm{CCD}$ camera

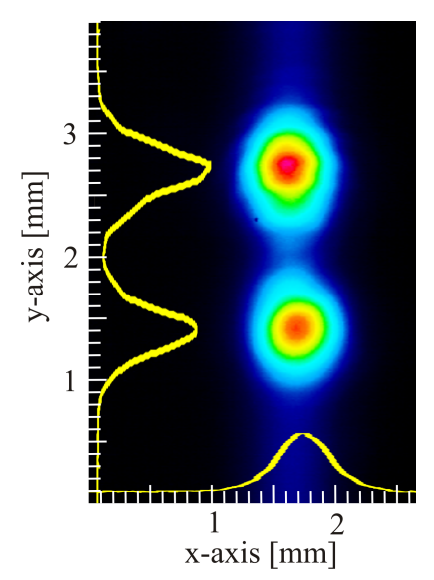

Fig. 6. Spatial output profile of the Q-switch output for the $17 \%$ OC with average output power of $50 \mathrm{~mW}$ at a distance of $150 \mathrm{~mm}$ from the OC showing the characteristic double spot.

\section{B. Laser output characterization}

Using a $\mathrm{T}=17 \%$ OC and a side-pump power of $48 \mathrm{~W}$, active Qswitching delivering an average output power of $50 \mathrm{~mW}$ was obtained when the MEMS mirror was operated at a mechanical resonance frequency of $7.905 \mathrm{kHz}$ providing a TOSA of $63^{\circ}$. In this case, the pump power value was limited to avoid potential damage to the micromirror gold coating due to excessive heat arising from the absorbed intracavity power in the coating layer. Two distinct output beams could be observed, as displayed by the transverse intensity profile taken using a CCD camera with an integration time larger than $50 \mathrm{~ms}$ and at a distance of $150 \mathrm{~mm}$ away from the OC (Fig. 6). The beam quality factor of both beams was measured to be identical $\left(\mathrm{M}^{2}=1.1\right)$. Using a faster detector, both beams were shown to emit in a sequential manner (i.e. the pulse train of both beams had an identical pulse repetition frequency (PRF) albeit delayed by half the period). The temporal behavior of both output beams including their combined pulse train, actuation signal of the micromirror and the cavity alignment crossing by the micromirror is shown in Fig. 7. One output pulse was

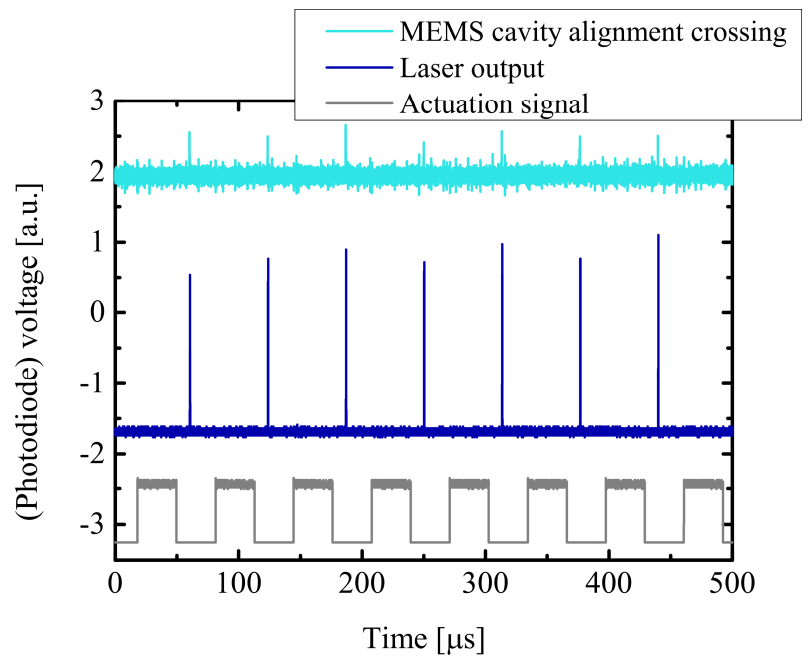

Fig. 7. Pulse train, cavity alignment crossing by the MEMS and voltage actuation signal for the cavity using the $17 \%$ OC and an average output power of $50 \mathrm{~mW}$. 


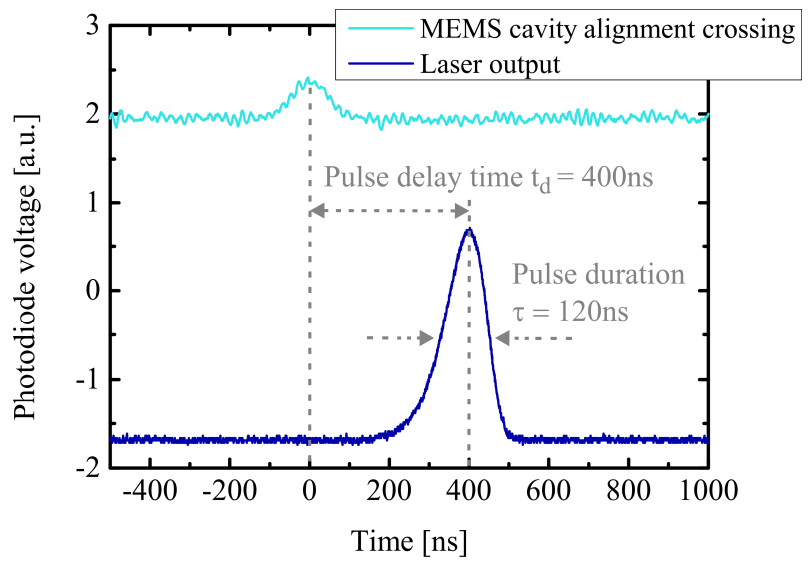

Fig. 8. Single output pulse and MEMS cavity alignment crossing signal, including the graphical definitions for the pulse delay time $t_{d}$ and pulse duration $\tau$ for the cavity using the $17 \%$ OC and an average output power of $50 \mathrm{~mW}$.

emitted as the scanning mirror passes through the cavity alignment. This resulted in the emission of two output beams with pulses of similar pulse duration per full mechanical movement cycle. Consequently, the PRF of each beam corresponded to the mechanical resonance frequency of the scanning micromirror.

The dual beam transverse output profile is induced by the pulse build-up time, which delays the pulse emission. Since the mirror rotates throughout this build-up time, the pulse is emitted at an angle in respect to the cavity alignment. The bidirectional movement of the scanning micromirror leads to the emission of this dual beam pattern, whereby a pulse delay time $t_{d}$ between the optimum cavity alignment crossing and pulse emission could be measured. For the case of an average output power of $50 \mathrm{~mW}$, using the $\mathrm{T}=17 \% \mathrm{OC}$, this delay time was measured at 400ns as shown in Fig. 8. The shape of the emitted pulses is also displayed with a full-width-halfmaximum pulse duration of $120 \mathrm{~ns}$. The resulting pulse energies and peak powers were $3.6 \mu \mathrm{J}$ and $26 \mathrm{~W}$ respectively. The pulse timing and amplitude jitters were measured to be $15 \mathrm{~ns} \pm 8 \mathrm{~ns}$ and around $25 \%$ respectively. It is believed that they mainly originate from pump fluctuations and from an oscillation jitter of the micromirror discussed in section II.A. Further discussion of this dual beam behavior, using the theoretical model, will be shown in section IV.

To evaluate the temporal and spatial characteristics of the output beams for varying gain settings of the side-pumped Nd:YAG module, the laser cavity from Fig. 4 was repeatedly built using four OCs with transmission values ranging from $41 \%$ to $10 \%$. In each case, the cavity alignment coincided with the axis running through the midpoint of the gain medium, leading to identical pulse durations and pulse delays for output pulses from both micromirror movement directions. The threshold for $\mathrm{cw}$ laser actuation was obtained at a pump power of $96 \mathrm{~W}, 66 \mathrm{~W}, 48 \mathrm{~W}$ and $37 \mathrm{~W}$ for these cavities, using a $41 \%$, $29 \%, 17 \%$ and $10 \%$ OC respectively. The resulting Qswitched pulse durations and pulse delay times delivering an average output power of $25 \mathrm{~mW}$ are shown in Fig. 9(a) and Fig.

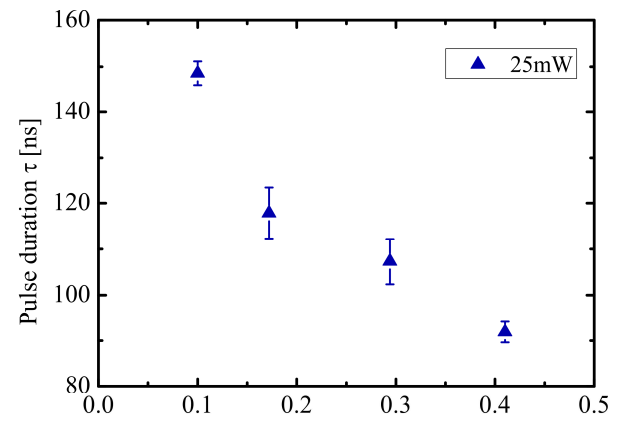

(a)

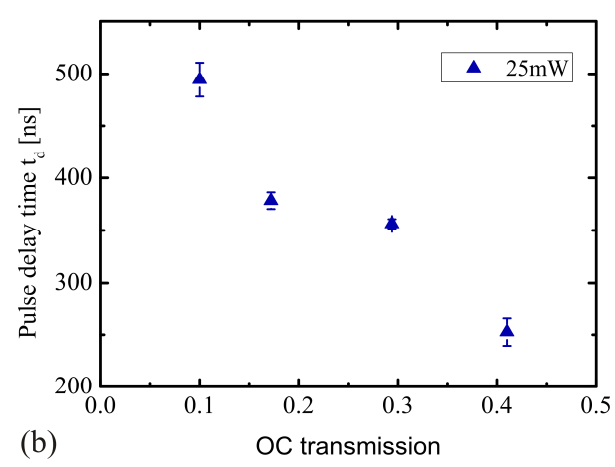

Fig.9. Variation of (a) the pulse duration $\tau$ and (b) the pulse delay time $t_{d}$ for the four OC transmissions and average output power settings of $25 \mathrm{~mW}$.

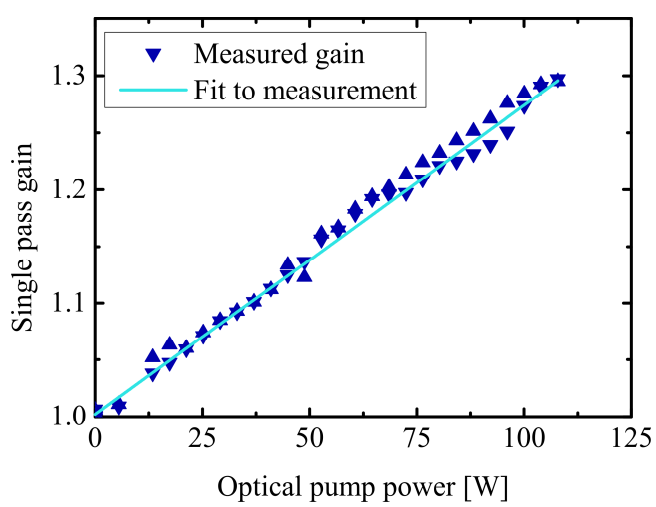

Fig. 10. Single pass gain measurement of the Nd:YAG pump module.

9(b) respectively. As expected, both time values rise with decreasing OC transmission and therefore reduction of the optical pump power. The pulse delay time ranged from $250 \mathrm{~ns}$ for the cavity using the $41 \%$ OC to $490 \mathrm{~ns}$ for the cavity using the $10 \%$ OC, with a pulse duration ranging from $90 \mathrm{~ns}$ to $150 \mathrm{~ns}$ for the respective cavities.

The minimum possible pulse duration for a given laser cavity can be estimated using [18]:

$\tau_{c} \approx 8.1 \cdot \frac{t_{r}}{\ln (G)}$

where $t_{r}$ is the cavity round-trip time and $G$ the unsaturated round-trip gain. A $500 \mathrm{~mW} \mathrm{cw} \mathrm{Nd}: \mathrm{YVO}_{4}$ probe laser at $\lambda=1064 \mathrm{~nm}$ was used to determine the single pass gain at the center of the Nd:YAG rod, which is displayed in Fig. 10. For the four cavity configurations described above, this leads to 


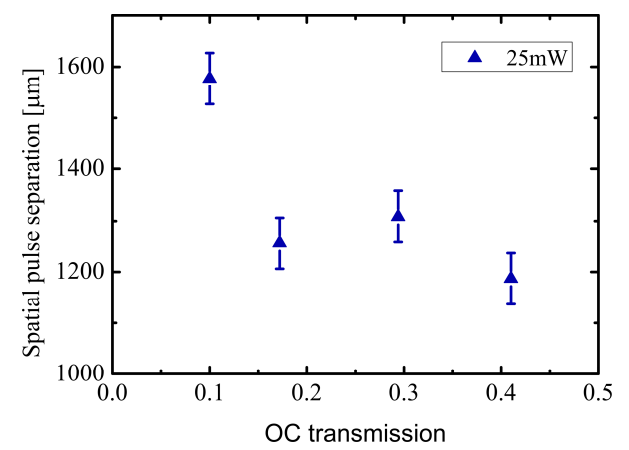

Fig. 11. Variation of the spatial distance between the two transverse output spots with changing OC transmission, measured at a distance of $150 \mathrm{~mm}$.

round-trip gains of $1.52,1.34,1.23$ and 1.16 , respectively, and subsequently to minimum pulse durations of $60 \mathrm{~ns}, 80 \mathrm{~ns}, 120 \mathrm{~ns}$ and $160 \mathrm{~ns}$, respectively. Since these calculated values are in the same order of the measured values, we can conclude that these MEMS micromirrors are efficient active Q-switches in these types of solid-state laser cavities.

Finally, the respective transverse midpoint separation between both output beams on a plane situated $150 \mathrm{~mm}$ away from the OC was measured in all four cases and is shown in Fig. 11. This measurement was repeated at several planes leading to the calculation of an angle between each spot in respect to the cavity midpoint axis of less than $0.5 \mathrm{mrad}$, with a converging tendency between both spots. In the case of the $17 \%$ OC, a slight decrease of the pulse delay time can be observed (Fig. 9(b)). This led to a reduced spatial distance between both beams as seen in Fig. 11. Such a trend cannot be fully explained yet but we believe that, in this case, the cavity alignment produced slightly higher intracavity losses $\left(\gamma_{0}\right)$ than in the other cases. In these lasers, this would lead to an increase of the ratio between the pump power delivering $25 \mathrm{~mW}$ of output power and the pump power at $\mathrm{cw}$ threshold (expressed as $\mathrm{k}$ in equation (5)). The simulation described in the next section will demonstrate that this will ultimately lead to a reduction of the pulse delay time.

\section{THEORETICAL MODEL OF SCANNING MICROMIRROR Q-SWITCH LASER DYNAMICS}

In this section, the laser rate equations are applied to our cavities including the experimentally measured dynamic loss induced by the micromirror rotation. The results are then presented and discussed.

\section{A. Analytical rate equation model}

The analytical simulation model describing the temporal laser output behavior in a Q-switched regime is based on solving the dynamic laser rate equations, similar in its basics to the work presented by Midwinter [13] and Lukac [14] on bulk rotating mirror Q-switches. The rate equations describing the temporal variation of the inversion population density $\mathrm{n}$ and the photon density $\phi$ can be written in the form [19]:

$$
\begin{aligned}
& \frac{d n}{d t}=-n \sigma \phi c \\
& \frac{d \phi}{d t}=\phi\left(\frac{l_{a}}{l_{c}} n \sigma c-\frac{\gamma(t)}{t_{r}}\right) .
\end{aligned}
$$

where $\sigma$ is the stimulated emission cross-section, $\mathrm{c}$ the speed of light in the gain medium, $1_{\mathrm{a}}$ the length of the gain medium, $1_{\mathrm{c}}$ the length of the overall laser cavity, $\gamma(\mathrm{t})$ the time-dependent loss per cavity round-trip and $t_{r}$ the cavity round-trip time. The time-dependent loss can be described by:

$\gamma(t)=\ln \left(\frac{1}{R_{O C}}\right)+\gamma_{0}+\gamma_{M E M S}(t)$.

and includes the losses induced by the $\mathrm{OC}$ reflectivity $\mathrm{R}_{\mathrm{OC}}$, the intrinsic optical losses $\gamma_{0}$ at perfect alignment of the MEMS micromirror and the time-dependent losses $\gamma_{\text {MEMS }}$ due to a misalignment of the cavity, originating from the rotation of the scanning micromirror. In the case of AOMs or EOMs-based Q-switching, this time-dependent contribution is usually approximated by a step function, while in our case, a description of the time dependent loss function is required. In previous work on bulk rotating mirror Q-switches, this timedependent function has been described in the form of a cosinefunction [13] or a linear fit to measured values around the mirror midpoint position [14]. The angular-dependent loss contribution in our laser cavities will be experimentally determined in the following sub-section.

The ordinary differential equation system expressed in (2) and (3) is solved using a Matlab-based Runge-Kutta method. In this numerical approach, the boundary conditions of the initial population inversion density $\mathrm{n}_{0}$ and initial photon density $\phi_{0}$ are required to describe the system before pulse emission. With no cavity alignment at this point, $\phi_{0}=1$ can be assumed which represents the optical noise from which a pulse build-up occurs. The value of $\mathrm{n}_{0}$ can be estimated using the ratio of the pump power $\mathrm{P}_{\mathrm{p}}$ and the pump power at threshold for $\mathrm{cw}$ output $\mathrm{P}_{\mathrm{pc}}$ combined with the critical inversion density at threshold $\mathrm{n}_{\mathrm{ic}}[20]$ :

$n_{0}=\frac{P_{p}}{P_{p c}} \cdot n_{i c}$

with the pump ratio $\mathrm{k}$ defined as $\mathrm{k}=\mathrm{P}_{\mathrm{p}} / \mathrm{P}_{\mathrm{pc}}$ and $\mathrm{n}_{\mathrm{ic}}$ as

$$
n_{i c}=\frac{-\ln R_{o c}+\gamma_{0}}{2 \sigma l_{a}}
$$

\section{B. Angular dependent cavity loss determination}

To determine the angle dependent loss function $\gamma_{\text {MEMS }}(t)$ required for solving equation (4), a Caird-analysis [21] was performed for a range of mirror tilt angles. The Caird-analysis uses the relationship between the slope efficiency $\eta_{\mathrm{s}}$ and 


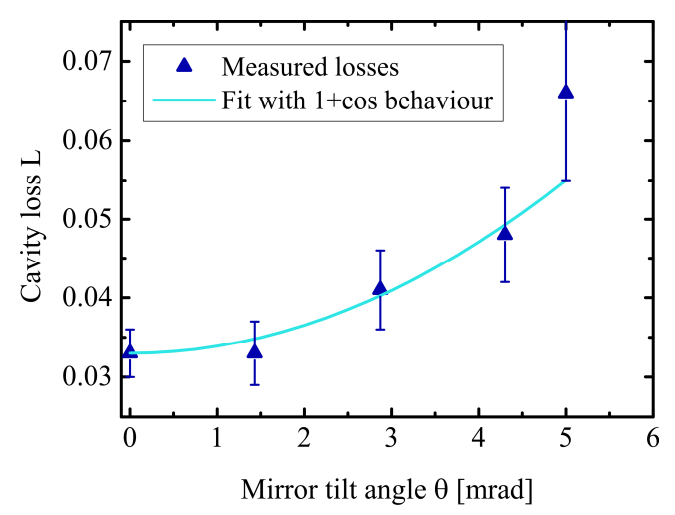

Fig. 12. Measurement of the angular dependent cavity losses using a Cairdanalysis with an HR equivalent mirror. (N.B. the reduction in accuracy at the $5 \mathrm{mrad}$ angle is due to the limited pump power range available)

output coupling fraction $\mathrm{T}$ to determine the cavity losses in the form

$$
\eta_{s}^{-1}=\eta_{0}^{-1}\left(1+L T^{-1}\right)
$$

where $\eta_{0}$ is the limiting slope efficiency in the absence of passive losses and $\mathrm{L}$ the combination of $\gamma_{0}+\gamma_{\text {MEMS. }}$. To ensure accurate measurement of the slope efficiency, output powers in excess of $1 \mathrm{~W}$ are required and could lead to micromirror damage. Consequently, a HR bulk mirror with similar surface curvature to that of the micromirror $(\mathrm{ROC}=250 \mathrm{~mm})$ was placed in a gimbal mirror mount. The rotation center of the mount was placed at the position of the MEMS micromirror it was replacing in the cavity.

The resulting determined loss values for mirror tilt angles between $0 \mathrm{mrad}$ and $5 \mathrm{mrad}$ are shown in Fig. 12, including a fit to the measured values. This fit defined the loss when the micromirror was perfectly aligned, $\gamma_{0}$, as 0.33 while the dynamic losses can be expressed as:

$$
\gamma_{M E M S}(t)=1+\cos (A \cdot \theta(t)+\pi)
$$

where $\mathrm{A}$ is a fitting parameter to the measurements $(\mathrm{A}=42$ in this case) and $\theta(t)$ originates from the bi-directional movement of the mirror and can be written as:

$$
\theta(t)=\theta_{\max } \cos (2 \pi f t),
$$

where $f$ is the mechanical resonance frequency of the micromirror and $\theta_{\max }$ the maximum amplitude of the mechanical mirror scan angle.

\section{Simulation model results}

To numerically solve the rate equation model, start and end times for the simulation are required. These values refer to the time points at which the micromirror allows pulse propagation through the gain medium. In these laser cavities, these values correspond to time points taken about $680 \mathrm{~ns}$ before and after

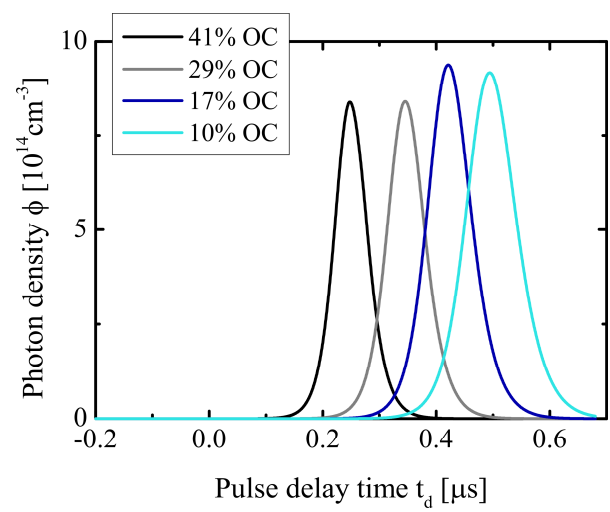

Fig. 13. Simulated photon density for the laser cavity configurations with $25 \mathrm{~mW}$ average output power presented in the experimental part.

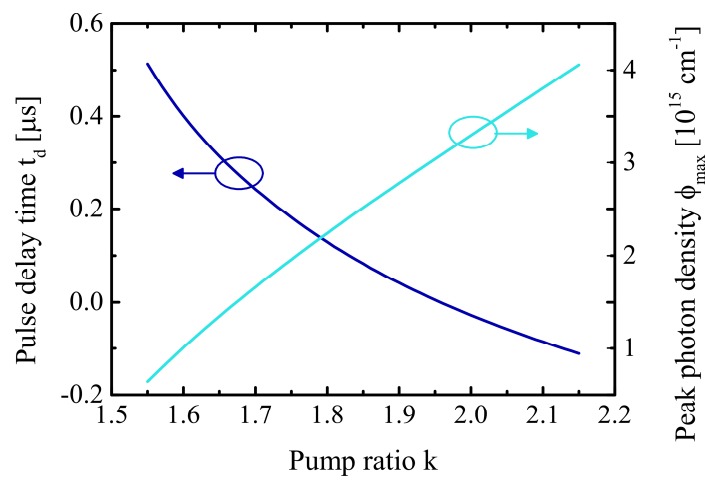

Fig. 14. Simulated pulse delay time and peak photon density with variation of the pump power ratio for the cavity using an OC with $\mathrm{T}=17 \%$.

the passage of the mirror normal incidence angle through optimum cavity alignment. Numeric simulations were performed for the four laser cavities described in the previous section. The different output coupling values resulted in different pump ratios $\mathrm{k}$ due to the associated variation in laser thresholds and slope efficiencies. The resulting intracavity photon densities temporal distribution for the four cases are shown in Fig. 13, with the zero position of the pulse delay time set when the mirror is at optimum cavity alignment. The pump ratios $\mathrm{k}$ were measured at $1.3,1.39,1.59$ and 1.82 , for the cavities using the $41 \%, 29 \%, 17 \%$ and $10 \%$ OC respectively. As expected, this increasing trend for $\mathrm{k}$ is due to a reduction of the laser conversion slope efficiency.

This numerical calculation was also performed in the case of the $17 \%$ OC cavity, delivering a $50 \mathrm{~mW}$ average output power. The variation of the pulse delay time and peak photon density are shown in Fig. 14 for increasing pump power ratios. An increase in $\mathrm{k}$ results in shorter pulse delay times due to the increased initial population inversion density. Consequently, the pulse emission at the exact point of the micromirror crossing through the cavity alignment could be obtained for a value of $\mathrm{k}=1.95$. At this point, the two output beams would be perfectly overlapped, leading to the emission of a single output beam with twice the PRF of one of the dual beam outputs. For higher pump ratios, the pulse delay times will turn negative leading to a change in the beam association with one of the 


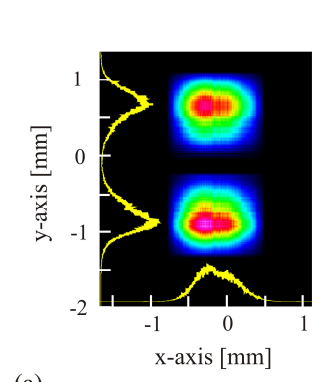

(a)

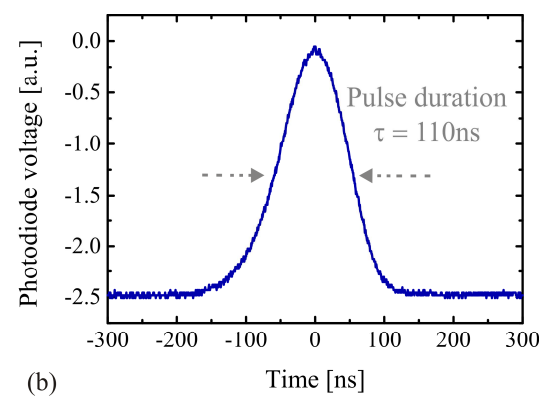

Fig. 15. Laser output using a dielectric coated micromirror with $650 \mathrm{~mW}$ average output power showing (a) the transverse output profile at a distance of $100 \mathrm{~mm}$ from the $17 \%$ OC and (b) the pulse profile with a duration of $110 \mathrm{~ns}$.

mirror rotation directions. Further increasing the pump ratio will ultimately lead to multiple pulsing per mirror scan.

\section{POWER-SCALING USING DIELECTRIC COATED MICROMIRRORS}

To increase the laser efficiency and reduce any thermallyinduced deformation of the micromirror surface, a HR dielectric coating was applied to the MEMS micromirror. This coating consisted of 9-pairs of $\lambda / 4$-thick $\mathrm{Nb}_{2} \mathrm{O}_{5}$ and $\mathrm{SiO}_{2}$ layers deposited using ion-assisted electronic beam deposition. In order to reduce any stress-induced deformation of the micromirror surface, this process consisted of four deposition steps separated by a full venting of the reaction chamber. In this way, surface deformation was minimized and the resulting concave curvature was measured at $\mathrm{ROC}=0.2 \mathrm{~m}$. Using a probe laser beam at $\lambda=1064 \mathrm{~nm}$, the reflectivity of the mirror surface was measured at $\mathrm{R}=98 \%$. In addition, this coating deposition resulted in a slight variation of the mechanical resonance frequency of the micromirror (now measured at $\mathrm{f}=7.93 \mathrm{kHz}$ ) but without any noticeable impact to the maximum scanning angle.

This dielectric-coated micromirror was inserted in the cavity described in Fig. 4, using the $17 \%$ OC and a MEMS cavity arm elongated by $10 \mathrm{~mm}$. Stable Q-switched oscillation of the MEMS laser was obtained with average output powers of up to $650 \mathrm{~mW}$ for $55 \mathrm{~W}$ of side-pump power. Again, the dual beam emission described in section III could be observed (see Fig. 15(a)). The shape of a typical pulse is shown in Fig. 15(b) with a duration of $110 \mathrm{~ns}$, which is comparable to the cavity limit estimated from [18]. The resulting pulse energies and peak powers are $41 \mu \mathrm{J}$ and $370 \mathrm{~W}$ respectively. Thermallyinduced instabilities of the MEMS resonance movement due to the absorption of the laser light within the silicon chip limited the maximum output power and had an impact on the transverse intensity profile as shown in Fig. 15(a).

\section{DISCUSSION}

To ensure the long term stability shown by MEMS micromirror technology, it is important to devise an efficient thermal management strategy for intracavity laser applications. First and foremost, this strategy relies on the deposition of HR dielectric coatings, as described above, in order to minimize any light absorption within the silicon device. Laser conversion efficiency will also be significantly improved by the use of HR coatings ensuring $\mathrm{R}>99.5 \%$. Furthermore, active cooling using Peltier elements will also alleviate thermal buildup and enhance long-term stability of the micromirrors. It must also be noted that this thermal build-up will be avoided in the transparency range of silicon (i.e. $\lambda>1.4 \mu \mathrm{m}$ ).

The simulations based on the rate equation approach described in section IV, are in agreement with the experimental characterization of the solid-state laser. This simulation could be further improved by undertaking a full spatial simulation approach of the dynamics of both the micromirror behavior and the laser. For example, this could be based upon a Fourier expansion simulation of the em-field or upon a FEM simulation of the oscillating em-field inside the cavity. However, these approaches would require highly complex calculations while still relying on assumptions concerning the two-way interaction between the micromirror and the laser cavity.

\section{CONCLUSION}

The output characteristics of a Nd:YAG solid-state laser actively Q-switched by a MEMS scanning micromirror were presented. A dual beam output profile was observed and its origin discussed together with an investigation of the influence of varying laser cavity parameters.

Using gold-coated micromirrors, maximum average output powers of $50 \mathrm{~mW}$ were obtained with pulse delay times in the order of $400 \mathrm{~ns}$ and timing jitter of $\sim 15 \mathrm{~ns}$. A numerical rate equation model was also developed based on this laser. Finally, power-scaling of this Q-switch technique was achieved using dielectric coatings on the micromirror surfaces resulting in average output powers of up to $650 \mathrm{~mW}$ and pulse energies of $41 \mu \mathrm{J}$. Further investigations in improving the properties of dielectric coatings are ongoing to further powerscale this technique and obtain pulse emission with zero delay time (i.e. single output beam emission).

This low-cost, energy efficient, miniature Q-switching technique is driven by the rapid development of optical MEMS technology. Through this, independent multi-beam output from a single laser gain medium can also be achieved by using MEMS micromirror arrays. Therefore this method has the potential to impact laser engineering with industrial, medical and defence applications.

\section{REFERENCES}

[1] R. Bauer, W. Lubeigt, and D. Uttamchandani, "Dual Q-switched laser outputs from a single lasing medium using an intracavity MEMS micromirror array," Opt. Lett., vol. 37, no. 17, pp. 3567-3569, Aug. 2012.

[2] Editorial, "Photonics West 2014," Nat. Photonics, vol. 8, no. 4, pp. 265-265, Mar. 2014.

[3] A. Inoue, T. Komikado, K. Kinoshita, J. Hayashi, and S. Umegaki, "Deformable Mirror for Mechanical Q-Switching of Laser-DiodePumped Microchip Laser," Jpn. J. Appl. Phys., vol. 46, no. No. 42, pp. L1016-L1018, Oct. 2007.

[4] M. Fabert, A. Desfarges-Berthelemot, V. Kermène, A. Crunteanu, D. Bouyge, and P. Blondy, "Ytterbium-doped fibre laser Q-switched by a 
cantilever-type micro-mirror," Opt. Express, vol. 16, no. 26, pp. 22064 $22071,2008$.

[5] M. Fabert, A. Desfarges-Berthelemot, V. Kermène, and A. Crunteanu, "Temporal synchronization and spectral combining of pulses from fiber lasers Q-switched by independent MEMS micro-mirrors," Opt. Express, vol. 20, no. 20, pp. 22895-22901, Sep. 2012.

[6] V. Couderc, A. Crunteanu, M. Fabert, F. Doutre, F. El Bassri, D. Pagnoux, and A. Jalocha, "Picosecond pulse generation in a hybrid Qswitched laser source by using a microelectromechanical mirror," Opt. Express, vol. 20, no. 5, pp. 5524-5529, Feb. 2012.

[7] A. Crunteanu, D. Bouyge, D. Sabourdy, P. Blondy, V. Couderc, L. Grossard, P. H. Pioger, and A. Barthelemy, "Deformable micro-electromechanical mirror integration in a fibre laser Q-switch system," J. Opt. A, vol. 8, pp. 347-351, 2006.

[8] D. Bouyge, A. Crunteanu, V. Couderc, D. Sabourdy, and P. Blondy, "Synchronized Tunable Q -Switched Fiber Lasers Using Deformable Achromatic Microelectromechanical Mirror," IEEE Photonics Technol. Lett., vol. 20, no. 12, pp. 991-993, 2008.

[9] M. Fabert, V. Kermène, A. Desfarges-Berthelemot, P. Blondy, and A. Crunteanu, "Actively mode-locked fiber laser using a deformable micromirror," Opt. Lett., vol. 36, no. 12, pp. 2191-2193, Jun. 2011.

[10] Y.-A. Peter, H. P. Herzig, E. Rochat, R. Dandliker, C. Marxer, and N. F. de Rooij, "Pulsed fiber laser using micro-electro-mechanical mirrors," Opt. Eng. SPIE, vol. 38, no. 4, pp. 636-640, 1999.

[11] W. Lubeigt, J. Gomes, G. Brown, A. Kelly, V. Savitski, D. Uttamchandani, and D. Burns, "Control of solid-state lasers using an intra-cavity MEMS micromirror," Opt. Express, vol. 19, no. 3, pp. 2456-2465, Jan. 2011.

[12] A. A. Vuylsteke, "Theory of Laser Regeneration Switching," J. Appl. Phys., vol. 34, no. 6, pp. 1615-1622, 1963.

[13] J. E. Midwinter, "The theory of Q-switching applied to slow switching and pulse shaping for solid state lasers," Br. J. Appl. Phys., vol. 16, no. 8, pp. 1125-1133, Aug. 1965.

[14] M. Lukac, "Output energy characteristics of optimally pumped rotating mirror Q-switch lasers," IEEE J. Quantum Electron., vol. 27, no. 9, pp. 2094-2097, 1991.

[15] M. Marincek and M. Lukac, "Development of EM field in lasers with rotating mirror Q-switch," IEEE J. Quantum Electron., vol. 29, no. 8, pp. 2405-2412, 1993.

[16] MEMSCAP Inc., 12 Alexander Drive, Building 100, Research Triangle Park, NC 27709, USA. .

[17] L. Li, R. Li, W. Lubeigt, and D. Uttamchandani, "Design, Simulation, and Characterization of a Bimorph Varifocal Micromirror and Its Application in an Optical Imaging System," J. Microelectromechanical Syst., vol. 22, no. 2, pp. 285-294, 2013.

[18] J. J. Zayhowski, "Microchip lasers," Opt. Mater. (Amst)., vol. 11, no. 2 3, pp. 255-267, Jan. 1999.

[19] W. Koechner, Solid-State Laser Engineering, 4th ed. Berlin Heidelberg: Springer-Verlag, 1996.

[20] O. Svelto, Principles of Lasers, 5th ed. Boston, MA: Springer US, 2010.

[21] J. A. Caird, S. A. Payne, P. R. Staber, A. J. Ramponi, L. L. Chase, and W. F. Krupke, "Quantum electronic properties of the $\mathrm{Na}_{3} \mathrm{Ga}_{2} \mathrm{Li}_{3} \mathrm{~F}_{12}: \mathrm{Cr}^{3+}$ laser," IEEE J. Quantum Electron., vol. 24, no. 6, pp. 1077-1099, Jun. 1988.

Ralf Bauer received the Dipl.-Ing. degree in Mechatronics from the University of Erlangen-Nuernberg, Germany in 2010, and the Ph.D. degree from the University of Strathclyde, Glasgow, U.K., in 2013 for work on MEMS micromirrors as active intracavity devices in solid-state lasers.

$\mathrm{He}$ is currently a Post-doctoral Research Associate in the Centre for Microsystems and Photonics, University of Strathclyde, working on the integration of MEMS devices in miniature photoacoustic spectroscopy gas sensors and optical sensors for trace gas detection. His research interests include the development and integration of MEMS in optical systems and laser systems.

Dr. Bauer is a member of the Optical Society and former vice-president and current committee member of the University of Strathclyde student chapter of the OSA, IOP and EPS.
Alan Paterson was born near Glasgow, Scotland in 1990. He received the MEng degree in electronic and electrical engineering from the University of Strathclyde, Glasgow, Scotland in 2013.

Currently he is undergoing a $\mathrm{PhD}$ project at the University of Strathclyde involving the integration of micro-electro-mechanical systems into solid-state laser systems as Q-switch elements.

Caspar Clark received the BSc (Hons) in microelectronics / physics from University of Paisley, Scotland in 1995 and Ph.D. degree in laser physics from Heriot-Watt University, Scotland in 2011.

He is currently the Chief Technical Officer of Helia Photonics Ltd having held engineering positions in defence and telecoms companies. He specialises in design, development and growth of high optical fluence resistant thin films for semiconductor devices. His research interests include active thin films and novel nanofabrication of metamaterials.

Deepak Uttamchandani (SM'05) received the Ph.D. degree from University College London, London, U.K., in the area of optical fiber sensors, in 1985.

$\mathrm{He}$ is currently the Head of the Centre for Microsystems and Photonics, University of Strathclyde, Glasgow, U.K. His early research in MEMS concentrated on optothermal microresonator sensors and in investigating techniques for general MEMS material characterization using MEMS micromechanical resonators. His recent research has concentrated on system applications of optical MEMS including intracavity MEMS-based laser systems, MEMS-based directional microphones and MEMS-based singlepixel imaging systems. He has also published in the field of optical sensors including subwavelength tip-based Raman spectroscopy, which has contributed to the development of tip-enhanced Raman spectroscopy and in the area of in situ intraocular drug detection systems via optical spectroscopy in the living eye.

Walter Lubeigt received the Engineering Diploma degree in opto-electronic systems from the Ecole Supérieure des Procédés Electroniques et Optiques, University of Orléans, France in 2001. He then received the PhD. degree from the University of Strathclyde, Glasgow, U.K. in 2006 for work on solid-state laser performance enhancement using intracavity adaptive optics techniques.

He subsequently worked on the development of diamond Raman lasers at the Institute of Photonics, University of Strathclyde. In 2010, he joined the Centre for Microsystems and Photonics, University of Strathclyde, as a John Anderson Research Lecturer. His current research interests include the development of MEMS-controlled solid-state lasers, the use of intracavity adaptive optics to improve the performance of solid-state Raman lasers and the development of novel laser systems for environmental remote sensing. 